\title{
Arbor
}

\section{Alimentos irradiados}

Sendra, E., Capellas, M. y Guamis, B.

Arbor CLXVIII, 661 (Enero 2001), 129-153 pp.

La industria alimentaria y las administraciones públicas realizan grandes esfuerzos para mejorar el mantenimiento de las condiciones higiénicas y evitar la contaminación de alimentos. Pese a estos esfuerzos siguen produciéndose un gran numero de procesos patológicos relacionados con los alimentos. Las buenas prácticas higiénicas pueden reducir el nivel de contaminación, pero algunos microorganismos patógenos resulta imposible eliminarlos, especialmente de aquellos alimentos que se comercializan crudos y con procesado mínimo. La irradiación se presenta como un método de descontaminación posible para este grupo de alimentos, especialmente válido como método de descontaminación final.

Dosis de irradiación inferiores a 10 kGy (dependiendo del alimento $y$ condiciones) son efectivas para eliminar posibles patógenos no esporulados, inactivar parásitos, tratar especias, condimentos y otros ingredientes secos, fruta fresca y productos de cuarta gama (vegetales frescos, limpios y envasados, listos para consumo).

Respecto a la seguridad de los alimentos irradiados, está plenamente demostrado que no provoca la aparición de compuestos especiales y provoca una ligera reducción en el contenido de algunas vitaminas, que es equiparable a la producida por otros tratamientos tecnológicos. Se ha visto también que microorganismos que han sido sometidos a radiaciones y han sobrevivido a ellas se muestran más sensibles a las condiciones ambientales de estrés que aquellos que nunca han sido irradiados.

Pese a que los organismos internacionales FAO y OMS presentan la irradiación como un método seguro, eficaz, limpio con el medio ambiente $y$ energéticamente eficiente, los consumidores siguen estando desinformados y mostrándose reticentes a su utilización. En la actualidad se están llevando a cabo muchos sondeos, de los que se deriva que una correcta in- 
formación al consumidor es el único método válido para conseguir la aceptación de esta tecnología.

Las investigaciones actuales en este sector se centran en la optimización de su aplicación a alimentos concretos y en los posibles efectos sobre los materiales de envasado. Por lo que respecta a la aplicación industrial, se tiende a crear centros de tratamiento donde diferentes productores llevan sus materias a tratar. Se espera que esta tecnología presente una fuerte tendencia creciente en los próximos años.

\section{Antecedentes}

El descubrimiento de los rayos X por W.K. Röetgen en 1895 y el descubrimiento en 1896 de las sustancias radioactivas por $\mathrm{H}$. Becquerel condujo al estudio de los efectos biológicos de estas radiaciones. El interés por la irradiación de alimentos resurgió a mediados de los años 40 cuando Huber sugirió el uso de aceleradores de electrones para la conservación de los alimentos, pero todavía eran costosos y de difícil aplicación en la práctica industrial. En los años 50 el interés se decantó por los isótopos radioactivos, con la previsión de un futuro de abundancia de tales sustancias.

Según cálculos estimados, las pérdidas anuales de granos y legumbres son de un $10 \%$ y las de verduras y frutas frescas de un $50 \%$ (OMS/FAO, 1989). Estas pérdidas son mayores en países en vías de desarrollo, favorecidas por las condiciones climáticas. Otro problema a tener en cuenta es la gran incidencia de las enfermedades de transmisión alimentaria, se calcula que su apreciación es de sólo un 5\% del total (Mossel, 1990). Estos han sido los motivos principales que han llevado a las grandes organizaciones internacionales -Organización de las Naciones Unidas para la Agricultura y la Alimentación (FAO), Organización Mundial de la Salud (OMS) y Agencia Internacional de Energía Atómica (AIEA) - a promover, primero, estudios sobre la irradiación de alimentos y, en segundo lugar, a proponer su uso para aquellas aplicaciones que han considerado necesarias (FAO/OMS, 1977, 1979, 1983, OMS/FAO, 1989, AIEA/FAO, 1992 y OMS, 1994). 


\section{Alimentos irradiados}

De todo esto no se puede deducir que la irradiación de los alimentos sea la panacea y no puede sustituir a la higiene en la manipulación ni a todos los tratamientos convencionales. Es una tecnología con un lugar propio dado que permite tratar alimentos para los que no hay otra alternativa.

La definición del estado actual de la irradiación pasa por la falta de armonización legislativa entre los diferentes países del mundo, como en el caso de la UE, donde no hay consenso entre los estados miembros (en Reino Unido y Alemania no está permitido), esto conlleva dificultades en el comercio internacional de alimentos irradiados (Loaharanu, 1989 y Derr, 1993). En el estado español está permitida desde 1966 (D.2725/1966 B.O.E. 31.10.66). La Unión Europea propuso la irradiación de alimentos como tema prioritario de estudio dentro del programa FLAIR (EUR 15017 EN). A partir de los resultados obtenidos por los expertos se ha iniciado un nuevo intento de regulación común de la irradiación de alimentos para la UE. El 22 de febrero de 1999 se adoptaron las directivas 1999/2/CE y 1999/3/CE relativas a la aproximación de las legislaciones de los Estados miembros sobre alimentos e ingredientes alimentarios tratados con radiaciones ionizantes y al establecimiento de una lista comunitaria de estos productos irradiados (D.O.C.E. L 66/16 13/3/99 y D.O.C.E. L 66/24 13/3/99). La única categoría que aparece en la lista es la de hierbas aromáticas secas, especias y condimentos vegetales, con un valor máximo de la dosis total de radiación absorbida de $10 \mathrm{kGy}$. La directiva establece que los Estados miembros de la UE no podrán prohibir la comercialización de los productos irradiados de esta categoría, sin perjuicio de las restricciones nacionales no relativas a dichos productos, que podrán seguir aplicándose. Con fecha límite del 31 de diciembre de 2000, se presentará una propuesta de lista positiva para completar la actual.

Por otra parte, en los Estados Unidos la autoridad sanitaria en materia de alimentación, la Food and Drug Administration (FDA) consideró, en 1958, las fuentes de radiaciones ionizantes como aditivos alimentarios. Así, se hacía necesario un estudio previo de seguridad como requisito para que la FDA aceptara un tratamiento. También era necesario un estudio previo a la salida del producto al mercado. De este modo, la irradiación se ha ido aprobando en USA tan sólo para aplicaciones específicas. La información solicitada por la FDA para establecer la seguridad de un alimento irradiado es la siguiente (Olson, 1998):

1. Seguridad radiológica: es decir, posible presencia de radioactividad inducida en los alimentos. 
2. Seguridad toxicológica: si existe evidencia de formación de tóxicos, qué parámetros o compuestos deben ser evaluados y qué tests pueden proporcionar información útil. Este segundo punto es muy difícil de evaluar pues la base del tratamiento es la absorción de energía y no la adición química. Suele llevarse a cabo alimentando animales de laboratorio con alimentos irradiados, aplicando factores de seguridad y de variabilidad individual y posteriormente extrapolando a humanos. Está aceptado que la aplicación de dosis de hasta 4 kGy no necesita test toxicológico pues nunca se ha observado toxicidad. Aquellos alimentos de gran consumo que vayan a tratarse a dosis elevadas sí requieren un estudio individualizado.

3. Seguridad microbiológica: si existe posibilidad de aparición de mutantes más virulentos o si puede darse el caso que la irradiación disminuya el número de microorganismos alteradores dejando a los patógenos crecer libres de competencia. Farkas (1989) observó que, al contrario de lo que sugieren estos postulados, la irradiación disminuía la virulencia y la adaptación a condiciones de estrés de las formas bacterianas normales. Este punto ya no supone una preocupación para la FDA. De todos modos sí se intenta asegurar la destrucción de Clostridium botulinum e impedir la producción de toxina.

4. Adecuación nutricional: si hay pérdida significativa de nutrientes, si el alimento a tratar supone una fuente importante de dicho nutriente. En definitiva se pretende asegurar que el aporte vitamínico no se vea afectado en la dieta global. Respecto a la pérdida de vitaminas, a veces la cocción resulta más destructiva que la irradiación.

La FDA y el USDA tienen aprobado el tratamiento de especias, frutas, vegetales, trigo y harina de trigo, carne de cerdo, pollo y carnes rojas (IFT, 1999; Anon., 2000). Existe además una demanda continua de aprobación de la irradiación, así, en agosto de 1999, la Food Irradiation Coalition, un grupo de 30 industrias alimentarias, organizaciones de salud, académicas y consumidores, solicitaron a la FDA la aprobación de la irradiación de carne y productos avícolas preparados para su consumo, frutas y vegetales, incluyendo semillas, frutos secos y brotes germinados. Basan su petición en que la pasterización fría que ellos persiguen ha sido ampliamente estudiada y se ha demostrado segura y efectiva. La aprobación del tratamiento de semillas germinadas ha sido solicitada por separado por otra industria (Mermelstein, 1999b). Asimismo, la creación de nuevas plantas de tratamiento, generalmente creadas por un grupo de industrias, va en aumento (Melmerstein, 1999a). 


\section{Alimentos irradiados}

\section{Definición, fundamento y fuentes}

La irradiación de alimentos es el proceso controlado para tratar alimentos con radiaciones ionizantes. La energía que éstos reciben es suficiente para romper enlaces químicos que provocan cambios en sus componentes y contaminantes. Los productos de la irradiación pueden estar eléctricamente cargados (iones) o ser neutros (radicales libres). Estos a su vez reaccionan dando lugar a cambios en el material irradiado que se conocen como radiolisis. Estas reacciones son las responsables de la destrucción de microorganismos, insectos y parásitos.

Las fuentes de radiaciones ionizantes autorizadas para alimentos son de dos tipos:

1) Fuentes mecánicas. Son equipos eléctricos, que a su vez se clasifican según las radiaciones emitidas:

1.1) Equipos generadores de rayos $X$.

1.2) Equipos generadores de electrones acelerados.

Las fuentes mecánicas ofrecen la ventaja de su seguridad, pues cuando no están en funcionamiento la emisión de radiaciones cesa, por lo que no revisten peligro alguno para los operarios ni para el medio que les rodea (Boaler, 1984).

2) Sustancias radioactivas. Las que pueden emplearse para tratar alimentos son el Cobalto 60 y el Cesio 137. El Parlamento Europeo propuso la desautorización del Cs-137 en 1989 (D.O.C.E. N.C. 291/58 20.11.89) dado que este isótopo se obtiene como producto secundario de los reactores nucleares y tiene una larga vida. Está permitido en Estados Unidos (FDA, 1987). Las radiaciones gamma que emiten las sustancias radioactivas tienen un poder de penetración adecuado para el tratamiento de la mayor parte de los alimentos. Tienen en su contra el hecho de que el radionucléido sigue desintegrándose continuamente, sin que se pueda detener el proceso de emisión aunque no estén en funcionamiento las plantas de tratamiento (Fellows, 1988 y Couvercelle-Halbwachs, 1989).

Las unidades en que se mide la energía de las radiaciones ionizantes son los electronvoltios (eV) siendo su equivalencia $1 \mathrm{eV}=1,610^{-19}$ julios. La unidad de dosis absorbida se denomina Gray (Gy) y se define como la energía media comunicada por la radiación ionizante a la materia por unidad de masa ( 1 gray $=1$ julio/kg). El Council for Agricultural Science and Technology estima que esta dosis rompe menos de 10 enlaces químicos por cada 10 millones de enlaces presentes (Olson, 1998). 
Sendra, E., Capellas, M. y Guamis, B.

En 1983 la Comisión FAO/OMS del Codex Alimentarius estableció una dosis máxima recomendada de $10 \mathrm{kGy}$. Una dosis de $10 \mathrm{kGy}$ es una cantidad muy pequeña de energía, equivalente a la cantidad de calor necesaria para elevar la temperatura del agua $2,4^{\circ} \mathrm{C}$ (OMS/FAO, 1989). La OMS en 1994 concluyó que la irradiación de alimentos no conlleva la formación de compuestos tóxicos, no eleva el riesgo microbiológico y no genera pérdidas de nutrientes que puedan afectar al estado nutricional de la población. En 1997 el comité conjunto de expertos de FAO-OMS-AIEA concluyó que los alimentos irradiados son seguros y salubres a cualquier dosis (OMS, 1997). La FDA debe aprobar ahora los materiales de envase para alimentos que van a ser irradiados.

El tratamiento de alimentos con radiaciones ionizantes con las fuentes mencionadas y a las dosis recomendadas no da lugar a radioactividad inducida en los alimentos (Fellows, 1988, Furuta et al. 1988 y Thayer, 1994). Además, no se han encontrado compuestos radiolíticos diferentes de compuestos formados por otros tratamientos (Crawford and Ruff, 1996).

\section{Aplicaciones en alimentos}

Algunas posibles aplicaciones son:

\section{Desinfestación}

En el proceso de desarrollo de los insectos, los huevos son la forma más sensible a las radiaciones y las formas adultas las más tolerantes pues aunque quedan estériles siguen viviendo y alterando el substrato un cierto tiempo.

Desinfestación de granos y harinas. A dosis de 0,75-1,5 kGy se obtienen resultados eficientes y sin aumento en la concentración de aflatoxinas en granos y harinas (Mitchell, 1988). Los insectos supervivientes no desarrollan radiorresistencia. En cambio se ha observado un aumento de ácidos grasos libres en harinas irradiadas, así como ligeras pérdidas de vitaminas A y tiamina, que se ven potenciadas si el tratamiento de irradiación va seguido de cocción (Thayer, 1990 y Rayas Duarte y Rupnow, 1994). En arroz irradiado se observaron pérdidas de algunas vitaminas: un $22 \%$ de tiamina, riboflavina, niacina y piridoxina (Murray, 1983). Aunque a las dosis de aplicación comercial no se observan cambios organolépticos, se conoce que los efectos de 


\section{Alimentos irradiados}

la irradiación en el almidón pueden dar cambios de comportamiento en sus derivados (Basson, 1983 y Ohinata et al. 1988).

Desinfestación de especias, vegetales deshidratados y frutos secos. La radiación a dosis de $3-10 \mathrm{kGy}$ se ha mostrado efectiva para el tratamiento de especias, condimentos, preparados enzimáticos y otros ingredientes secos (Farkas, 1998). Una dosis de 0,3 kGy es suficiente para erradicar la mayoría de plagas de insectos en frutos secos, en general dosis de hasta $0,9 \mathrm{kGy}$ no ocasionan cambios nutricionales $\mathrm{y}$ de calidad de consideración, sólo los frutos secos con alto contenido en ácidos grasos insaturados empiezan a presentar efectos adversos a partir de 0,6 kGy (Johnson y Marcotte, 1999). En plantas medicinales, aplicando dosis de $10 \mathrm{kGy}$ en manzanilla, no se encontraron variaciones en los aceites esenciales y compuestos hidrofílicos extraídos de flores irradiadas (Katusin-Razem et al. 1985). En cacao, no se observaron cambios organolépticos en los derivados obtenidos de cacao irradiado (Appiah et al. 1982). En vegetales deshidratados para sopas, se observó un ligero aumento de la viscosidad y una disminución del tiempo de ebullición de la sopa, se vio que $3 \mathrm{kGy}$ era la dosis óptima, sin dar lugar a cambios organolépticos (Paul et al. 1969).

Desinfestación, retraso de la maduración y alteración de la fruta fresca. Estos efectos se consiguen a las mismas dosis. En este caso se pueden dar pérdidas de algunas vitaminas (niacina, tiamina, riboflavina y beta-caroteno), poco importantes para las dosis comerciales de aplicación en estos alimentos. El ácido ascórbico pasa a dehidroascórbico, que también tiene efecto de vitamina. Los azúcares irradiados en presencia de oxígeno pueden dar lugar a azúcares con dos grupos carbonilo. Se recomienda una dosis de 0-1 kGy para la desinfestación de fruta fresca (Thayer, 1990). En estudios con aguacates, irradiando después de la descontaminación en baño de agua caliente y el envasado, se observa una mejora en el mantenimiento de la calidad (Ang et al. 1986). En manzanas 'Delicious', se ha visto que la dosis óptima de irradiación es de $2 \mathrm{kGy}$, duplicando su vida útil (Lastarria Tapia y Sequeiros, 1985). En frutas tropicales está recomendado como tratamiento de cuarentena (Thomas, 1986 y AIEA/FAO, 1992).

\section{Inhibición de la aparición de brotes en bulbos y tubérculos}

En patatas, la dosis está limitada a 0,15 kGy debido a la aparición de pardeamiento (Tasumitsu et al., 1972, 1974). En cebollas se ha visto que hay una inhibición total de brotes y una disminución de pérdidas por deshidratación y podredumbre (Matin et al. 1985). 


\section{Inactivación de parásitos}

Se ha estudiado la inactivación de helmintos y protozoos en carnes y pescados. Este efecto se consigue a bajas dosis. Como ejemplo, las dosis de inactivación para las especies más importantes son: Toxoplasma gondii, 0,25 kGy; Cysticercus bovis y Cysticercus cellulosae, 0,4-0,6 kGy y Trichinella spiralis $0,3 \mathrm{kGy}$. A estas dosis sólo se han encontrado pérdidas de un 5-18\% de tiamina al irradiar carne de cerdo (Engel et al., 1988 y Thayer, 1990). En general, dosis de 0,15 a 0,7 kGy en determinadas condiciones de tratamiento son efectivas para la inactivación de parásitos y garantizan que el consumo de estos alimentos es seguro (Farkas, 1998).

\section{Eliminación de bacterias patógenas}

Este efecto se ha estudiado en carnes rojas, de aves, pescados y mariscos. Dosis de 2 a $7 \mathrm{kGy}$ (dependiendo del alimento y condiciones) pueden eliminar posibles patógenos no esporulados presentes en alimentos, incluyendo: Salmonella spp, Staphylococcus aureus, Campylobacter, Listeria monocytogenes y Escherichia coli O157:H7. (Farkas, 1998), sin modificar sus propiedades sensoriales, nutricionales y tecnológicas. En este caso, la dosis está limitada por los cambios organolépticos. No se puede utilizar la irradiación para sustituir totalmente la sal, nitratos y nitritos pero sí en combinación con ellos con lo que se permite la reducción de dosis. En estudios realizados en carne de pollo se concluyó que la irradiación prolongaba el tiempo de vida útil de 2 a 4 semanas (Cho et al. 1985). La carne de ave tiene su dosis máxima de aplicación en 2,5 kGy. Dosis superiores a ésta causan cambios de sabor, para no rebasarla y mejorar las condiciones sanitarias se recomienda combinar la irradiación con el uso de atmósferas modificadas (Lambert et al. 1991 y Grandison y Jennings, 1993). También en carnes rojas se han observado muy buenos resultados a dosis bajas para el control de patógenos (Thayer, 1993).

\section{En legumbres}

En legumbres se han obtenido buenos resultados. Como ejemplo, la aplicación de dosis de 2,5 a $10 \mathrm{kGy}$ disminuye el tiempo de cocción y aumenta la terneza de las legumbres cocidas (Barbosa-Cánovas et al. 1998).

En soja irradiada con dosis entre 2.5 y $10 \mathrm{kGy}$ para la posterior obtención de licuado y tofu, se observó una disminución del tiempo 
de cocción y de remojo dando como consecuencia un mayor rendimiento. En cambio dosis superiores afectaron negativamente a las proteínas de soja (Myung-Woo et al. 1994).

\section{En productos cárnicos}

Los productos cárnicos constituyen el último grupo de alimentos de reciente aprobación para su tratamiento por radiaciones. Los microorganismos alteradores más comunes son Gram negativos psicrótrofos que, a su vez, son muy susceptibles a la irradiación pues se eliminan prácticamente con dosis de $1 \mathrm{kGy}$ (Monk et al. 1995). En cambio, tienen muy poco efecto sobre Gram positivos productores de ácido (Thayer et al. 1993). La calidad de la carne puede verse más o menos afectada, dependiendo de la dosis, temperatura y atmósfera durante el tratamiento y de las condiciones de almacenamiento. En carne cruda, pueden desarrollarse olores extraños que desaparecen al cocinar ( $\mathrm{Lu}$ chsinger et al. 1996). Pueden producirse también cambios de color: irradiando en ausencia de oxígeno se acentúa el brillo de los colores (Lebepe et al. 1990), mientras que en presencia de oxígeno se producen decoloraciones (Grant y Patterson, 1991a). Irradiando a bajas temperaturas y en ausencia de oxígeno se minimizan los cambios en la carne. Para asegurar la higiene de las carnes irradiadas éstas deben ser envasadas antes del tratamiento.

\section{En productos de la pesca}

Los estudios realizados en pescado y productos de la pesca han dado muy buenos resultados, habiéndose encontrado diferentes niveles de descontaminación en función de las dosis utilizadas (Ninjoor et al. 1981, Maha et al. 1981, Giddings, 1984 y Hammad y El-Mongy, 1992). Se han observado pérdidas de la vitamina tiamina poco importantes a dosis de $1 \mathrm{kGy}$, con la que se obtiene una disminución importante de microorganismos sin afectar a las características organolépticas (Ming-Sai Liu et al. 1991). Para especies subtropicales se recomienda el tratamiento a $1 \mathrm{kGy}$ como óptimo por las características sensoriales y de conservación que se obtienen, mientras que el límite máximo de dosis es de 3 kGy debido a la aparición de cambios organolépticos indeseables (Poole et al. 1994). Idénticos resultados se han obtenido más recientemente para merluza (Silva et al. 1994). Se ha visto que al irradiar gambas en estado congelado se evita la aparición de malos olores (Ito et al. 1987, 1988). 
Sendra, E., Capellas, M. y Guamis, B.

\section{Esterilización de alimentos}

Básicamente destinados a la alimentación de animales de laboratorio (Thayer, 1990).

\section{Irradiación de queso}

Aplicada al queso Camembert de leche cruda para permitir su exportación a USA (Fiess, 1992 y Boissseau, 1994).

\section{Irradiación como alternativa a algunos aditivos}

El uso de radiaciones ionizantes puede sustituir el uso de desinfestantes: profam/clorofam en patatas, hidracida maleica en cebollas, fosfina o bromuro de metilo en granos y legumbres, dibromuro de etileno en fruta fresca, óxido de etileno en especias, condimentos, hierbas aromáticas... También puede sustituir el uso de antimicrobianos como ácido benzoico y cítrico en gambas y camarones, y reducir la dosis de nitritos necesaria en productos curados.

La irradiación puede ser una alternativa al uso de algunos aditivos químicos pero no es seguro que vaya a serlo (Diehl, 1992). El paso de la posibilidad de aplicación a su uso comercial dependerá de la necesidad real, en los casos siguientes: para evitar pérdidas de alimentos que no tienen otra alternativa de tratamiento, en alimentos perecederos de elevado coste económico y donde la irradiación permite un mejor mantenimiento de la calidad frente a otros tratamientos (Hammad y El-Momgy, 1992) y en la esterilización de alimentos para pacientes inmunosuprimidos (Hashisaka et al. 1990) o preparados de administración parenteral (Koornhof et al. 1994).

\section{Efecto sobre los alimentos y sus contaminantes. Limitaciones de aplicación}

\section{Formación de radicales}

Un radical libre se define como una especie química capaz de existir de forma independiente que contiene uno o más electrones desapareados (esto es, un electrón que ocupa un orbital atómico o molecular sólo). La vida media de los radicales que se forman por efecto del tratamiento con radiaciones es de $10^{-9}$ segundos y se calcula que bajo un tratamiento de $10 \mathrm{kGy}$ se forman $5 \times 10^{-3}$ moles de radicales por kilo de alimento (Grootveld et al. 1990 y Fellows, 1988). 


\section{Alimentos irradiados}

Los compuestos formados son predecibles a partir de la composición del alimento e idénticos a los que se forman por otras vías (Basson, 1983, Elias, 1989 y Grootveld et al. 1990) como son la acción de enzimas (p.e. lipooxigenasas, peroxidasas y xantina oxidasa), la oxidación de la grasa y ácidos grasos (Lebovics et al. 1992) y la degradación de vitaminas liposolubles y pigmentos.

Los efectos principales que producen en los alimentos consisten en: hidroxilaciones aromáticas de los fenoles y ácidos fenólicos -interesante por la formación de O-tirosina por hidroxilación de la fenilalanina-, cambios oxidativos en el ADN con rotura de hebras dobles y simples y formación de productos volátiles a partir de la degradación de ácidos grasos, que se pueden estudiar por cromatografía de gases (Grootveld et al. 1990).

\section{Efectos nutricionales}

Existe un estudio comparativo del efecto de la irradiación con rayos gamma y con electrones acelerados en los principales nutrientes frente a la congelación y el tratamiento térmico a elevada temperatura, así como del efecto de la alimentación exclusiva con carne de pollo así tratada sobre diferentes especies animales. De los resultados obtenidos se deriva que los efectos que estos tratamientos tienen en los alimentos y sus efectos nutricionales son comparables para las distintas tecnologías estudiadas (Thayer, 1990).

Las proteínas, las grasas, los hidratos de carbono, los minerales y los elementos traza no se ven afectados a dosis de 10 kGy. Las vitaminas son los nutrientes más sensibles y pueden afectarse dependiendo del sustrato, dosis, temperatura y presencia de oxígeno durante el tratamiento (Diehl, 1995). Según datos de la OMS las condiciones para la pérdida mínima de vitaminas son: bajas temperaturas, ausencia de oxígeno y envasado adecuado del alimento. Las vitaminas hidrosolubles por orden de mayor a menor sensibilidad son: tiamina, ácido ascórbico, piridoxina, riboflavina, ácido fólico, cobalamina y ácido nicotínico. Las liposolubles, en el mismo orden: $\mathrm{E}$, caroteno, A, K y D (OMS, 1994). Cuando se valoran las pérdidas de vitaminas con cualquier tratamiento tecnológico hay que considerar la magnitud de la pérdida o reducción y la importancia del alimento en concreto en la dieta de la población como fuente de dicha vitamina. Así por ejemplo, los alimentos que constituyen las principales fuentes de vitaminas $\mathrm{A}$ y $\mathrm{E}$ en nuestra dieta (lácteos, yema de huevo, mantequilla, grasas y aceites) no son buenos candidatos a ser tratados por radiaciones por los cambios 
indeseables que se presentan (Diehl, 1991,1992 y Bloomfield, 1993). A su vez, la vitamina A y sus precursores son más radiosensibles en estado puro que en un sistema físico-químico complejo como puede ser un alimento (Bloomfield, 1993).

Por todo ello, diversos investigadores así como el Comité mixto de expertos FAO/AIEA/OMS concluyen que la irradiación de alimentos a dosis inferiores a $10 \mathrm{kGy}$ no causa cambios nutricionales importantes (Diehl et al. 1991a, 1991b, Thayer, 1990, Murray, 1983 y OMS/FAO, 1989).

\section{Efectos en la microbiota}

Los microorganismos irradiados supervivientes quedan con poca vitalidad, no habiéndose detectado aumento en la radio-resistencia ni aparición de mutaciones que den lugar a nuevos microorganismos. Los efectos microbiológicos son comparables a los que causa la aplicación de los procesos habituales de conservación de alimentos (Teufel, 1983). En general, las bacterias Gram negativas son más sensibles a las radiaciones que las Gram positivas y todas ellas más sensibles que las formas esporuladas de bacterias, los mohos y los virus (Couvercelle-Halbwachs, 1989) (Tabla 1).

TABLA 1. Dosis de reducción decimal $\left(\mathrm{D}_{10}\right)$

\begin{tabular}{|c|c|c|c|}
\hline Microorganismo & Dosis (kGy) & Producto & Referencia \\
\hline Aeromonas hydrophyla & $0,14-0,19$ & carne de vacuno & 1 \\
\hline Aeromonas hydrophila & 0,05 & gambas congeladas & 2 \\
\hline Campylobacter yeyuni & 0,19 & volatería & 1 \\
\hline Escherichia coli O157:H7 & 0,27 & carne de vacuno & 1 \\
\hline Listeria monocytogenes & 0,77 & carne de pollo & 1 \\
\hline Salmonella spp. & $0,38-0,77$ & carne separada mecánicamente & 1 \\
\hline Staphylococcus aureus & 0,36 & carne separada mecánicamente & 1 \\
\hline Clostridium botulinum $^{\mathrm{a}}$ & 3,56 & carne de pollo congelada & 1 \\
\hline Vibrio parahaemolyticus & 0,35 & gambas congeladas & 2 \\
\hline Pseudomonas spp. & 0,06 & - & 3 \\
\hline Lactobacillus spp. & 0,15 & - & 3 \\
\hline Micrococcus spp. & 0,70 & - & 3 \\
\hline Streptococcus fecalis & $0,5-1$ & - & 3 \\
\hline Micrococcus radiodurans & 8 & - & 3 \\
\hline
\end{tabular}

Referencias: (1) Thayer, 1993, (2) Rashid et al. 1992, (3) Couvercelle-Habwachs, 1989. a esporas 


\section{Alimentos irradiados}

La eficacia bactericida de una dosis dada de irradiación depende de: tipo y especies de microorganismos, recuentos iniciales, condiciones en que se encuentran los microorganismos, condiciones ambientales $(\mathrm{pH}$, temperatura, composición química de los alimentos, presencia o ausencia de oxígeno) y estado físico del alimento durante el tratamiento (Barbosa-Cánovas et al. 1998).

Estudios en Escherichia coli enterohemorrágica han revelado que la resistencia inducida al medio ácido confiere resistencia a la radiación, este hecho debe tenerse en cuenta para calcular el valor de reducción decimal $\mathrm{D}$, es decir, el tiempo en minutos necesario para inactivar el 90\% de la población de un microorganismo a una dosis determinada (Buchanan et al. 1999). El valor D aumenta de 0,22 a 0,31 para las células adaptadas al medio ácido (Buchanan et al. 1998).

Respecto a las temperaturas de aplicación de radiaciones, las especies Escherichia coli, Campylobacter jejuni y Salmonella, inoculadas en carne de vacuno picada, pueden inactivarse a dosis inferiores a $1 \mathrm{kGy}$ y en todos los casos el valor de reducción decimal $D$ es inferior al irradiar en refrigeración que al hacerlo en congelación $\left(-15\right.$ a $\left.-17^{\circ} \mathrm{C}\right)$ (Clavero et al. 1994).

Las toxinas microbianas en general y las aflatoxinas en concreto son estables a la irradiación (Lambert et al. 1991 y Kume et al. 1987). En cuanto a los hongos responsables de su formación, se ha observado que especies del género Aspergillus irradiadas experimentaban un aumento de su capacidad productora de aflatoxinas en medios de cultivo enriquecidos (Teufel, 1983) pero esta capacidad no se modifica cuando el sustrato es un alimento (Mitchel, 1988 y Borsa et al. 1992). En estudios realizados con el género Penicillium se observó una reducción de la capacidad productora de patulina por parte de $\mathrm{P}$. patulum irradiado (Bullerman and Hartung, 1975).

Cuando se ha estudiado el efecto de la irradiación sobre componentes puros se ha visto que los cambios inducidos son mayores que cuando se irradia una matriz más compleja como es un alimento (BarbosaCánovas et al. 1998).

La irradiación puede eliminar los microorganismos alteradores pero no enmascarar el mal olor y aspecto de un alimento ya alterado. Las dosis generalmente recomendadas para alimentos no permiten la destrucción total de microorganismos. Una desventaja frente a los tratamientos térmicos es que no se inactivan las enzimas y por tanto siempre que se pueda convendría combinar con un tratamiento de escaldado. La mayor potencialidad de la irradiación es su combinación con otros tratamientos, por ejemplo: tratamientos térmicos moderados, 
atmósferas modificadas o sales curantes, o para el tratamiento de zumo de naranja y cremogenado de mango con la adición de sorbato potásico (Barbosa-Cánovas et al. 1998).

\section{Limitaciones de aplicación y combinación de tratamientos}

No se pueden generalizar los efectos de las radiaciones ionizantes si no se especifican la dosis, condiciones y producto a tratar. La temperatura y la presencia o ausencia de oxígeno son determinantes de los efectos del tratamiento (Diehl, 1992). Las dosis comerciales son generalmente de pocos $\mathrm{kGy}$, dados los cambios que se producen en las características organolépticas de los alimentos. Los efectos organolépticos y nutricionales derivados de la aplicación de las radiaciones pueden minimizarse si se combina con otros tratamientos, lo que nos permite disminuir las dosis de ambos (Diehl, 1991 y Kilcast, 1991).

No todos los alimentos son buenos candidatos a ser tratados por radiaciones, de igual modo que no todos los tratamientos de conservación pueden aplicarse a todos los alimentos. Como ejemplo, algunas frutas se ablandan y decoloran, la viscosidad de la clara de huevo se altera y la hace inaceptable, la leche adquiere sabores desagradables. En muchos alimentos la dosis de tratamiento es limitada debido a las alteraciones organolépticas. La mayoría de frutas frescas y vegetales resisten un máximo de 2,25 kGy. Algunas alteraciones pueden evitarse irradiando en estado congelado (Barbosa-Cánovas, 1998).

Se acostumbra a combinar la irradiación con el uso de antioxidantes (Maha et al. 1981). Hay que considerar que algunas combinaciones con aditivos químicos conllevan modificaciones organolépticas (la adición de cloruro sódico, de $\mathrm{SO}_{2}$ y la disminución del $\mathrm{pH}$ ). Estas modificaciones pueden ser deseables o no según el alimento. En el caso de productos curados podemos disminuir la dosis necesaria de sales curantes a utilizar pero nunca sustituirlas (Diehl, 1992).

La combinación de la irradiación con el control de la temperatura $\mathrm{y}$ ambiente gaseoso durante el tratamiento y las condiciones adecuadas de almacenaje son los métodos más idóneos para minimizar los cambios que se producen al irradiar (Kilcast, 1991). Aunque cada alimento tiene sus condiciones óptimas concretas, en general se acepta que irradiar a temperaturas criogénicas y en ausencia de oxígeno es la combinación más recomendable (Diehl, 1991, Grant y Patterson 1991b, Kildcast, 1991, Lambert et al. 1991, Grandison y Jennings 1993 y Lebovics et al. 1994). 


\section{Alimentos irradiados}

La humedad del alimento y el ambiente durante el tratamiento influyen en la sensibilidad de los microorganismos a la irradiación: a mayor humedad relativa ambiental, mayor radio-resistencia presentan las esporas de Aspergillus flavus (Amoako et al. 1981). Los alimentos con bajo contenido en humedad mantienen durante más tiempo los productos de radiolisis, pero éstos disminuyen rápidamente al ser expuestos a la humedad ambiente o al entrar en contacto con el agua (Hayashi et al. 1972, 1973, Komiya y Nara, 1974 y Thayer, 1990). Para algunos alimentos puede resultar de utilidad controlar este parámetro durante el tratamiento.

Otras posibilidades son la combinación con tratamientos de inactivación de enzimas (Ninjoor et al. 1981) y con tratamientos térmicos por calor (Kaupert et al. 1981).

\section{Efecto en los materiales de envasado}

El efecto de la irradiación no es duradero a no ser que utilicemos el envase adecuado para preservar de la recontaminación (Diehl, 1992). A su vez el efecto que la irradiación pueda tener en los materiales de envase y embalaje debe ser estudiado (materiales, tintas y aditivos de envases: Kilcast, 1990, Allen et al. 1990 y Helle et al. 1993).

La FDA tiene pendiente de aprobación los materiales de envasado que pueden utilizarse. Hasta el momento, se han realizado estudios sobre posibles migraciones desde plásticos de polipropileno irradiados utilizados para la cocción al vacío y la conservación de carnes. Los compuestos que potencialmente pueden migrar del plástico al alimento son aditivos (menos del 1\% del total) y mayoritariamente oligómeros. Los estudios han mostrado que el tratamiento de los plásticos a dosis elevadas $(80 \mathrm{kGy})$ reduce el riesgo de migraciones frente al uso de bajas dosis de radiación (Marque et al. 1998).

Se ha observado que se produce migración de los aditivos dioctil adipato (DOA) y acetil tributil citrato (ATBC) - utilizados como plastificantes de PVC y PVDC/PVC - al aceite de oliva. Esta migración es mayor para el DOA y aumenta con la dosis, mientras que el ATBC no presenta migraciones a dosis inferiores a $20 \mathrm{kGy}$ (Goulas et al. 1998).

\section{Proceso y dosimetría}

El proceso de irradiación de alimentos consiste en exponer éstos a una fuente de energía de manera que absorban una dosis precisa. 
Para controlar el proceso es necesario conocer la producción de energía de la fuente por unidad de tiempo, conocer las características físicas del equipo (distancia fuente-producto) y exponer el alimento el tiempo necesario para que absorba la dosis deseada. La dosis a utilizar vendrá dada por el alimento a tratar y el efecto que queremos obtener (Tabla 2).

TABLA 2. Dosis recomendadas para algunos productos

\begin{tabular}{|c|c|c|c|}
\hline Alimento & Dosis (kGy) & Efecto & Referencia \\
\hline Cereales & $0,2-3$ & Desinfestación & Murray, 1983 \\
\hline Patatas & 0,075 & Inhibición de la germinación & Fiszer et al. 1985 \\
\hline Cebollas & $0,050-0,080$ & Inhibición de la germinación & Matin et al. 1985 \\
\hline Manzanas & 2 & Retraso de la maduración & $\begin{array}{l}\text { Lastarria-Tapia y } \\
\text { Sequeiros, } 1985\end{array}$ \\
\hline Carne fresca y pescado & $0,15-0,7$ & Eliminación de parásitos & Farkas, 1998 \\
\hline Carne Fresca $10-15^{\circ} \mathrm{C}$ & & Eliminación de & Grant y \\
\hline $25 \% \mathrm{CO}_{2} \quad 75 \% \mathrm{~N}_{2}$ & 1,75 & enterobacterias & Patterson, 1991b \\
\hline Camembert $5^{\circ} \mathrm{C}$ & 2,5 & Pasterización & Boisseau, 1994 \\
\hline $\begin{array}{l}\text { Pescado y productos de la } \\
\text { pesca } 2{ }^{\circ} \mathrm{C}\end{array}$ & $0.75-2.5$ & zación & \\
\hline Gamba congelada $20^{\circ} \mathrm{C}$ & $3,5-5$ & Destrucción de Vibrio & $\begin{array}{l}\text { Poole et al. } 1994 \\
\text { Rashid et al. } 1992\end{array}$ \\
\hline Especias y condimentos & $8-10$ & Descontaminación & Kume et al.1987 \\
\hline Plantas medicinales & 10 & Descontaminación & Katusim-Razem \\
\hline Nueces & $0,3-0,9$ & Desinfestación & $\begin{array}{l}\text { Johnson y Mar- } \\
\text { cotte. } 1999\end{array}$ \\
\hline $\begin{array}{l}\text { Preparados nutritivos para } \\
\text { administración parenteral }\end{array}$ & 1,5 & Pasterización & $\begin{array}{l}\text { Koornhof et al. } \\
1994\end{array}$ \\
\hline
\end{tabular}

La dosis máxima a utilizar vendrá limitada por el mantenimiento óptimo de la calidad del alimento irradiado (Camcigil, 1991).

En la literatura científica se encuentra una gran variedad de dosímetros para controlar el proceso, con pocas repeticiones, de lo que se deduce que el seguimiento de la dosis recibida no está estandarizado. Entre los trabajos en que se hace mención a la medición de dosis se encuentran: estudios de dosimetría (Sharpe, 1990), dosímetro opticrómico (Grandison y Jennings, 1993), dosímetro de Fricke (Rashid et al. 1992), film radiocrómico (Hashisaka et al. 1990) y calorímetro de agua (Brynjolfsson, 1989).

Actualmente, un tercio de las instalaciones existentes son multipropósito, son centros de tratamiento donde se utilizan diferentes 
fuentes según el nivel de penetración deseado (Barbosa-Cánovas et al. 1998).

Todas las instalaciones para irradiación de alimentos están obligadas a cumplir normas estrictas de seguridad. En la actualidad cada vez se tiende más al uso de equipos mecánicos (Brynjolfsson, 1989).

\section{Detección de alimentos irradiados}

Se necesitan métodos para la detección de los alimentos que han sido irradiados. Las autoridades gubernamentales los necesitan para verificar e inspeccionar, así como para regular el comercio internacional y la industria los necesita para su propio control de calidad (Camcigil, 1991). A su vez, la posibilidad de detección mejora la confianza del consumidor.

La detección de alimentos irradiados con el propósito de regular su aplicación es un activo campo de estudio. En 1993, Glidewell et $a l$. publicaron una interesante revisión del tema. Por el momento no se ha encontrado ninguna técnica aplicable a todos los alimentos y las más aceptadas son: detección de hidrocarbono y ciclobutanona en alimentos ricos en grasas, resonancia de spin electrónico para alimentos que contengan hueso y termoluminiscencia para alimentos que contengan silicatos minerales. Otras técnicas que se intenta desarrollar están basadas en la detección de daños en $\mathrm{ADN}$, valoración del daño celular y determinación de ratios entre células viables y totales.

No hay una huella universal del tratamiento por radiaciones y no es posible por tanto un método de detección universal. La detección de hidrógeno sería uno posible, siempre apoyando a otro complementario, pero posee limitaciones (Barbosa-Cánovas et al. 1998).

\section{Aceptabilidad de alimentos irradiados}

En los últimos años se han llevado a cabo un gran número de estudios sobre la aceptabilidad de alimentos irradiados por los consumidores, mayoritariamente en Estados Unidos. De entre los consumidores, los varones, a mayor grado de educación, y de entre ellos aquellos con mayores ingresos y residentes en áreas no urbanas constituían el grupo de población con mayor inclinación a consumir productos irradiados. La población de mayor edad y la de color expresó un mayor grado de desconfianza en estas prácticas de producción (Nayga, 1996). 
Los grupos con menores ingresos económicos y menor grado de estudios son los más preocupados. En general las mujeres se preocupan más que los hombres (Lusk et al. 1999). Al consumidor le cuesta entender por qué deben irradiarse los alimentos cuando el mismo alimento sin irradiar puede estar también presente en el mercado.

$\mathrm{Al}$ parecer, los consumidores están más preocupados por la seguridad de los operarios de las plantas de irradiación que por que los alimentos se vuelvan radioactivos tras el tratamiento. Los consumidores confiesan no conocer mucho sobre la irradiación de alimentos y es el concepto radiación lo que les asusta. Preferirían que se les presentase la irradiación como un proceso, no como aditivo, que es como lo contempla la FDA. Prefieren la palabra irradiación a radiación, a esta segunda le encuentran más connotaciones negativas y creen que en cuanto se familiaricen con el etiquetado, el logo de alimento irradiado llegará a ser suficiente. Piensan que es una tecnología muy nueva de la que no hay suficientes estudios y que no tiene todos los problemas solucionados, y tarde o temprano alguien puede cometer un error y algo puede salir mal. Desconocen que la irradiación, a las dosis permitidas, no induce radioactividad en los alimentos y que no provoca cáncer. Se debe tener especial cuidado de no dar la imagen que la irradiación puede sustituir a las medidas sanitarias apropiadas. La presencia de hormonas del crecimiento, pesticidas y residuos de tratamientos veterinarios en alimentos son los principales riesgos captados por el consumidor ( $>50 \%)$, mientras que la irradiación y la presencia de toxinas naturales de los alimentos son percibidos como alto riesgo por sólo un 26\% de los americanos. El 99\% de los americanos encuestados consideran que el correcto etiquetado de los alimentos es primordial. (Resurrección y Gálvez, 1999).

Los empresarios, por su parte, mantienen el deseo de ser los segundos en lanzarse y evitar dar el primer paso y reclaman ayudas para facilitar el etiquetado, como cambiar el nombre a "pasterización en frío» y que no fuera obligatorio indicar el símbolo de alimento irradiado (IFT, 1999).

Una consulta a dietistas de Estados Unidos sobre los posibles métodos para la reducción de enfermedades de transmisión alimentaria concluyó que preferían la correcta implantación de los sistemas de Análisis de peligros y puntos de control crítico que la irradiación o el empleo de aditivos químicos (Giavalma et al. 1998). Es necesario tener en cuenta la actitud de los grupos influyentes de profesionales y proporcionarles correcta información sobre el tema.

Desafortunadamente, las nuevas tecnologías (irradiación y biotecnología) están mediatizadas, un $87 \%$ de la población americana cree 


\section{Alimentos irradiados}

todo lo que los medios de comunicación dicen acerca de seguridad alimentaria (Sloan, 1999). La mayoría de estudiosos del tema coinciden en que, con el tiempo, el conocimiento de las ventajas económicas y la clara superioridad tecnológica de la irradiación para el tratamiento de algunos alimentos como el pollo y sus derivados, llevará a la aceptación de este proceso por el consumidor y que la correcta información, a ser posible proporcionada por autoridades sanitarias (Bruhn, 1995), prevalecerá sobre las distorsiones y desinformación originadas por los oponentes a la irradiación de alimentos (Andrews et al. 1998).

Las campañas informativas sobre los beneficios de la irradiación se han mostrado efectivas. Se han realizado estudios en supermercados en los que se observó que, sin información previa, un 51,5\% compraría carne de vacuno irradiada, pero que si se realizaba una campaña informativa este porcentaje crecía hasta un 71,3\% (Ressurrección y Gálvez, 1999).

Aunque en la UE se están llevando a cabo un gran número de estudios sobre irradiación de alimentos, no disponemos todavía de esta clase de estudios de mercado y no podemos saber cuál será la respuesta del consumidor medio europeo frente a la comercialización de alimentos irradiados. De entrada, es previsible que las reticiencias sean mayores en Europa, donde los hábitos culinarios son más tradicionales que en Estados Unidos. Además existen opiniones políticas sobre este tema (los grupos Verdes se declaran abiertamente en contra), los consumidores somos menos perceptivos a las innovaciones y tenemos un bajo conocimiento de las grandes pérdidas de alimentos que se producen en el mundo, generalmente en zonas lejanas de Europa.

\section{Bibliografía}

AIEA/FAO (1992): Use of irradiation as a quarantine treatment of food and agricultural commodities. Viena.

Anónimo (2000): USDA approves red meat irradiation. Meat Processing March/April 2000. p. 11.

Allen, D. W., Crowson, A., Leathard, D. A. y Smith, C. (1990): The effects of ionising radiation on additives present in food-contact polymers. En: Johnston, D. E. \& Stevenson, M. H. Food Irradiation and the Chemist. Conference. Cambridge: Royal Society of Chemistry, pp. 124-139.

Amoako AtтA, B., Odamtten, G. T. y Appiah, V. (1981): Influence of relative humidity on radiosensitivity of Aspergillus flavus. Infecting cocoa beans. Combination processes in food irradiation, pp. 161-168. 
Andrews, L.S., Ahmedna, M., Grodner, R.M., Liuzzo, J.A., Murano, P.S., Murano, E.A., Rao, R.M., Shane, S. and Wilson, P.W. (1998): Rev. Environmental Contamination and Toxicology, 154, 1-53.

Ang, L. A., Langerak, D. I., Duren, M. D. A., Uzcategu, E., Farkas, J. y Rubio, C. T. (1986): Comparative evaluation of untreated and radurized Chilean avocados shipped to the Netherlands. Acta Alimentaria 15(1), 57-67.

Appiah, V., Odamtten, G. T. y Langerak, D. I. (1982): The evaluation of some quality parameters of cocoa after the combination treatment of heat and radiation. Proceedings International Cocoa Research Conference 8, 777-781.

Barbosa-Cánovas, G.V., Pothakamury, U.R., Palou, E., and Swanson, B.G. 1998: Food Irradiation. En: Nonthermal preservation of foods. 161-213. Marcel Dekker Inc., New York, USA.

BAsson, R. A. (1983): Advances in radiation chemistry of food and food components. An overview. En: Elias, P.S. y Cohen, A.J.(Ed.), Recent advances in food irradiation. Amsterdam: Elsevier Biomedical Press., pp. 7-26.

Boisseau, P. (1994): Irradiation and the food industry in France. Food Tech. 5, 138-140.

Borsa, J., Chelack, W. S., Marquardt, R. R. y Frohlich, A. A. (1992): Comparision of irradiation and chemical fumigation used in grain desinfestacion on production of ochratoxin A by Aspergillus alutaceus in treated barley. J. Food Protect. 55(12), 990-994.

BoAler, V. J. (1984): Electron accelerator facilities for food processing. J. Food Engin. $3(4), 285-294$

Bloomfield, L. (1993): Food irradiation and vitamin A deficiency. Food Policy 2, 64-72.

BRUHN, C.M. (1995): Strategies for communicating the facts on food irradiation to consumers. Journal of Food Protection, 58(2), 213-216.

Brynjolfsson, A. (1989): Future radiation sources and identification of irradiated foods. Food Tech. 43(7), 84-89.

Buchanan, R.L., Edelson, S.G. Snipes, K. and Boyd, G. (1998): Inactivation of Escherichia coli O157:H7 in apple juice by irradiation. Applied Enviromental Microbiology, 64(11): 4533-4535.

Buchanan, R.L., Edelson, S.G. and Boyd, G. (1999): Effects of pH and acid resistance on the radiation resistance of enterohemorragic Escherichia coli. Journal of Food Protection, 62(3): 219-228.

Bullerman, L. B. y Hartung, T. E. (1975): Effect of low level gamma irradiation on growth and patulim production by Penicilium patulum. J. Food Sci. 40, 195.

Camcigil, M. (1991): Dose limits in food irradiation. Food Control 7, 130-132.

Cho, H. O., Lee, M. K., Byun, M. W., Kwon, J. H. y KIM, J. G. (1985): Radurization of the microorganisms contaminating chicken. Korean Journal of Food Science and Technology 17(3), 170-174.

Clavero, M.R., Monk, J.D., Beuchat, L.R., Doyle, M.P. and Brackett, R.E. (1994): Inactivation of Escherichia coli O157:H7, salmonellae and Campylobacter jejuni in raw ground beef by gamma irradiation, Applied Enviromental Microbiology, 60(6), 2069-2075.

Couvercelle-Halbwachs, C. (1989): L'ionisation des denrées alimentaires (I): Ann. Fals. Exp. Chim. 82(3), 159-168.

Crawford, L.M., y RuFf, E.H. (1996): A review of the safety of cold pasteurization through irradiation. Food Control 7(2), 87-97. 


\section{Alimentos irradiados}

DERR, D. D. (1993): International regulatory status and harmonization of food irradiation. J. Food Protect. 56(10), 882-886,892.

DiEHL, J. F. (1991): Nutritional effects of combining irradiation with other treatments. Food Control 1, 20-24.

DieHL, J. F., HASSELMAN, C. y KILCAST, D. (1991a): Nutritional value of irradiated foods. Medecine et Nutrition 27(5), 285-294.

Diehl, J. F., Hasselmann, C. y Kildcast, D. (1991b): Regulation of food irradiation in the European Community: is nutrition an issue? Food Control 9, 212-219.

DiEHL, J. F. (1992): Food irradiation: is it an alternative to chemical preservatives?. Food Additives and Contaminants 9(5), 409-416.

DieHL, J.F. (1995): Nutritional adequacy of irradiated foods. En: Safety of irradiated foods. 2nd Edición. Edd: Marcel Dekker, Inc, New York, NY.

ELIAS, P. S. (1989): New concepts for assessing the wholesomeness of irradiated foods. Food Tech. 43(7), 81-83.

Engel, R. E., Post, A. R. y Post R. C. (1988): Implementation of irradiation of pork for Trichina control. Food Tech. 42(7), 71-75.

FARKAS, J. (1989): Microbial safety of irradiated foods. Review. International Journal of Food Microbiology, 35(9), 1-15.

FARKAS, J. (1998): Irradiation as a method for decontaminating food. A review. International Journal of Food Microbiology, 44(3): 189-204.

FAO/OMS (1977): Salubrité des Aliments Irradiés. Rapport Comité mixte AIEA/FAO/OMS. Roma.

FAO/OMS (1979): Recommended international general standard for irradiated foods and recommended international code of practice for the operation of radiation facilities for the treatment of foods. FAO/WHO food standdars programme. Codex Alimentarius Comission.

FAO/OMS (1983): Inocuidad Microbiológica de los Alimentos Irradiados. Comisión del Codex Alimentarius. Roma.

FDA. (1987): Irradiation in the production, processing and handling of food. Code of Federal Regulations, part 179.

Fellows, P. (1988): Ambient temperature processing. Irradiation. En: Horwood, E. Food processing technology: principles and practice. Ellis Horwood Ltd., Chichester and VCH Verlagsgesellschaft mbH, Weinheim, pp.186-195.

Fiess, M. (1992): Isigny milite pour l'ionisation. RIA 474 du 20 Janvier ay 2 Février, 38-40.

Fiszer, W., Zabielski, J. y Mroz, J. (1985): Preservation of potatoes by irradiation and economic considerations. Food irradiation processing, pp. 101-108.

Foster, A. (1991): Consumer attitudes to irradiation. Food Control 1, 12-14.

Furuta, M., Katayama, T., Toratani, H. y Takeda, A. (1988): Preliminary examination of induced radioactivity in peppers by $10 \mathrm{MeV}$-electron irradiation. Food Irradiation 23(2), 93-99.

Giamalva, J.N., Redfern, M. and Bailey, W.C. (1998): Dietitians employed by health care facilities preferred a HACCP system over irradiation or chemical rinses for reducing foodborne diseases. Journal of American Dietitians Association, 98(8), 885-888.

Giddings, G. G. (1984): Radiation processing of fishery products. Food Tech. 38(4), 61-65,94-97. 
Glidewell, S.M., Deighton, N., Goodman, B.A. y Hillman, J.R. (1993): Detection of irradiated food: a review. Journal of the Science of Food and Agriculture, 61, 281-300.

Goulas, A.E., Riganakos, K.A., Ehlermann, D.A., Demertzis, P.G. and Kontominas, M.G. (1998): Effect of high-dose electron beam irradiation of DOA and ATBC plastizicers from food grade PVC and PVDC/PVC films, respectively, into olive oil. Journal of Food Protection, 61(6), 720-724.

Grandison, A. S. y JENNings, A. (1993): Extension of the shelf life of fresh minced chicken meat by electron beam irradiation combined with modified atmosphere packaging. Food Control 4(2), 83-88.

Grant, I. R. y Patterson, M. F. (1991a): Effect of irradiation and modified atmosphere packaging on microbiological and sensory quality of pork stored at refrigeration temperature. Int. J. Food Sci. Tech. 26, 507-519.

Grant, I. R. y Patterson, M. F. (1991b): Effect of irradiation and modified atmosphere packaging on the microbiological safety of minced pork stored under temperature abuse conditions. Int. J. Food Sci. Tech. 26, 521-533.

Grootveld, M., Jain, R., Claxson, W. D., Naughton, D. y Blake, R. D. (1990): The detection of irradiated foodstuffs. Trends in Food Science \& Technology 7, 7-14.

Hammad, A. A. I. y El-Mongy, T. M. (1992): Shelf-life extension and improvement ot the microbiological quality of smoked salmon by irradiation. J. Food Processing and Preservation 16, 361-370.

Hashisaka, A. E., Matches, J. R., Batters, Y., Hungate, F. P. y Dong, F. M. (1990): Effects of gamma irradiation at $-78^{\circ} \mathrm{C}$ on microbial populations in dairy products. J. Food Sci. 55(5), 1284-1289.

Hayashi, T., YANO, M. y NAMIKI, M. (1973): Formation and disappearance of radiationinduced radicals in foods. II. Rice and wheat. Food Irradiation 8(1), 95-99.

Hayashi, T., KaWakishi, S., NAmiki, M., NARA, S. y KomiYA, T. (1972): Formation and disappearance of radiation-induced radicals in foods. Food Irradiation 7(1), 15-19.

Helle, N., Schulzki, G., Linke, B., Spiegelberg, A., Boegl, K. W., Schreiber, G. A., Grabowsk, H. U. von, Pfordt, J., Maumrmann, U., Juelicher, S., Bischoff, C., Vater, N. y Heitmann, M. (1993): Identification of irradiated pasteurized egg products: a combined method to use in routine control. Zeitsch. Lebens. Unters. Forschung 197(4), 321-331.

IFT. 1999. Facts on food irradiation featured by the IFT New York Section. Food Technology, 53(1), 94-95.

Ito, H., Adulyatham, P. y Ishigaki, I. (1987): Distribution of bacteria in frozen shrimps and their decontamination by gamma irradiation. Food Irradiation 22(2), 19-22.

Ito, H., SANGthong, N. y Ishigaki, I. (1988): Effect of gamma-irradiation on frozen shrimps: inactivation of microorganisms and shelf-life extension of defrosted shrimps. Food Irradiation 23(2), 72-76.

Johnson, J. y Marcotte, M. (1999): Irradiation control of insect pests of dried fruits and walnuts. Food Technology, 53(6), 46-51.

Katusin Razem, B., Razem, D., Dvornik, I., Matic, S. y Minokovic, V. (1985): Radiation decontamination of dry chamomile flowers and chamomile extract. Food irradiation processing, pp. 69-77.

Kaupert, N. L., Lescano, H. G. y Kotliar, N.(1981): Conservation of apple and pear juice concentrates. Synergic effect of heat and radiation. Combination processes in food irradiation, pp. 205-216. 


\section{Alimentos irradiados}

KILCAST, D. (1990): Irradiation of packaged food. En: Johnston, D. E. \& Stevenson, M. H. Food Irradiation and the Chemist. Conference. Cambridge: Royal Society of Chemistry, pp. 140-152.

KILDCAST, D. (1991): Irradiation and combination treatments. Food Control 1, 6-8.

KomiYa, T. y NARA, S. (1974): The effects of moisture content on the formation of radicals in various starches by gamma-irradiation. II. Changes in radical contents of various irradiated starches in relation to adsorption and desorption of moisture. Food Irradiation 9(12), 62-68.

Koornhof, H.J., Isaacson, M., Saunders, J., van-Wyc, C.J., KotZe, A.F., Rosekilly, I.C. and DU PLESSIS, T.A. (1994): Enhancement of microbiological safety levels of aseptically admixed total parenteral nutrition solutions through low dose gamma irradiation. Journal Clinical Pharmacological Therapy, 19(4), 249-256.

Kume, T., Ito, H. y Ishgak, I. (1987): Production of aflatoxins and radiosensitivity of moulds isolated from spices. Food Irradiation 22(2), 16-18.

LAmbert, A. D., Smith, J. P. y DodDS, K. L. (1991): Combined effect of modified atmosphere packaging and low-dose irradiation on toxin production by Clostridium botulinum in fresh pork. J. Food Protect. 54(2), 94-101.

Lastarria Tapia, H. J. y Sequeiros, N. (1985): Effect of gamma-irradiation on apple var. "Delicious" stored under ambient or refrigerated conditions. Food irradiation processing, pp. 55-60.

Lebepe, N., Molins, R.A., Charoen, S.P., Iv, H.F. y Showronski, R.P. (1990): Changes in microflora and other characteristics of vacuum packaged pork loins irradiated at 3 kGy. Journal of Food Science, 55, 918-924.

Lebovics, V. K., GaÁl, Ö., Somogyi, L. y Farkas, J. (1992): Cholesterol oxides in gamma-irradiates spray-dried egg powder. J. Sci. Food Agric. 60, 251-254.

Lebovics, V. K., GAÁL, Ö., FARKAS, J. y SomogYI, L. (1994): Influence of packaging atmosphere on the formation of cholesterol oxides in gamma-irradiates egg powder. J. Sci. Food Agric. 66, 71-73.

LOAHARANU, P. (1989): International trade in irradiated foods: regional status and outlook. Food Tech. 43(7), 77-80.

Luchsinger, S.E., Kropf, D.H., García Zepeda, C. M., Chambers, M.E., Hollingsworth, M.E., Hunt, M.C., Marsden, J.L., Kastner, C.L. y Kuecker, W.G. (1996): Sensory analysis and consumer acceptance of irradiated boneless pork chop. Of Food Science, $61,1261-1266$.

Lusk, J.L., Fox, J.A. and Mcilvain, C.L. (1999): Consumer acceptance of irradiated meat. Food Technology, 53(3), 56-59.

Maha, M., Sudarman, H., ChosdU, R., Siagian, E. G. y Nasran, S. (1981): Combination of potassium sorbate and irradiation treatments to extend the shelf-life of cured fish products. Combination processes in food irradiation, pp. 305-318.

Marque, D., Feigenbaum, A., Dainelli, D. and Riquet, A.M. (1998): Safety evaluation of an ionized multilayer plastic film used for vacuum cooking and meat preservation. Food Additives and Contaminants, 15(7), 831-841.

Matin, M. A., Hossain, M. M., Amin, M. R., Rahman, S., Rokeya, B., Malek, M. A., Siddiqu, A. K. y Hossain, M. A. (1985): Pilot-scale studies on irradiation and storage of onions. Food irradiation processing, pp. 17-33.

Mermelstein, N.H. (1999a): Electron-Beam pasteurization plant near completion. Food Technology, 53(8), 88. 
Mermelstein, N.H. 1999b. Washington News. Food Technology, 53(9), 36.

Ming-Sai Liu, Ru-Yin Chen, Meei-Ju Tsaiy JuI-Sen YANG(1991): Effect of gamma irradiation on the keeping quality and nutrients of Tilapia (Oreochromis mossambicus) and Silver Carp (Hypophthalmichthys molitrix) stored at $1^{\circ} \mathrm{C}$. J. Sci. Food Agric. 57, 555-563.

Mitchell, G. E. (1988): Influence of irradiation of food on aflatoxin production. Food Tech. Australia 40(8), 324-326.

Monk, J.D., Beuchat, L.R. y Doyle M.P. (1995): Irradiation inactivation of food-borne microorganisms. Journal of Food Protection, 58, 197-208.

Mosher, P. (1992): Irradiation: in the consumer's hands. Citrus and Vegetable Magazine 56(1), 50-56.

Mossel, D. A. (1990): Irradiación de alimentos. Algunas medidas dirigidas a procurar la protección del consumidor frente a las infecciones entéricas alimentarias a lo largo de la cadena de producción de los alimentos de origen animal. Alimentaria 4, 45-51.

MurRay, T. K. (1983): Nutritional aspects of food irradiation. En: Elias, P.S. y Cohen, A.J.(Ed.), Recent advances in food irradiation. Amsterdam: Elsevier Biomedical Press., pp. 203-216.

Myung-Woo Byun, Kang I. J., Hayashi, Y., Matsumura, Y. y Mori, T. (1994): Effect of gamma irradiation on soya bean proteins. J. Sci. Food Agric. 66, 55-60.

NAYGA, R.M. (1996): Sociodemographic influences on consumer concern for food safety: the case of irradiation, antibiotics, hormones and pesticides. Review of Agricultural Economics, 18(3), 467-475.

Ninjoor, V., Doke, S. N. y NADKaRn, G. B. (1981): Storage stability and improved quality of fish products by enzyme suppression and gamma irradiation. Combination processes in food irradiation, pp. 319-333.

Ohinata, H., Muramatsu, N., OHARA, T., OHIKE, T. y ITo, H. (1988): Use of gamma-irradiation for microbial inactivation of buckwheat flour and buckwheat flour products. V. Studies on physical properties and texture of irradiated fresh-style soba noodle and on soba noodle processing properties of irradiated buckwheat flour. Food Irradiation 23(2), 11-20.

Olson, D.G. (1998): Irradiation of Food. Food Technology, 52(1), 56-62.

OMS/FAO (1989): La irradiación de los alimentos. Organización Mundial de la Salud. Ginebra.

OMS (1994): Safety and nutritional adequacy of irradiated food. Organización Mundial de la Salud. Ginebra.

OMS (1997): Food Irradiation press release WHO/68. Sept.19. Organización Mundial de la Salud. Ginebra.

Paul, N., Gruenewald, T. y Kuprianoff, J. (1969): Possibility of treating dry soups with ionizing radiation. Deutsche Lebensmittel Rundschau 65(9), 279-81.

Poole, S. E., Mitchell, G. E. y MaYze, J. L. (1994): Low dose irradiation affects microbiological and sensory quality of sub-tropical seafood. J. Food Sci. 59(1), 8587,105 .

RASHI, H. O., Iто, H. y IshigaKI, I. (1992): Distribution of the pathogenic vibrios and other bacteria in imported frozen shrimps and their decontamination by gammairradiation. World Journal of Microbiology and Biotechnology 8, 494-499.

Rayas Duarte, P. y Rupnow, J. H. (1994): Gamma-irradiated dry bean (Phaseolus vulgaris) starch: physicochemical properties. J. Food Sci. 59(4), 839-843. 


\section{Alimentos irradiados}

Ressureccion, A.V.A. and Galvez, F.C.F. (1999): Will consumers buy irradiated meat? Food Technology, 53(3),52-55.

ShARPE, P. H. G. (1990): Dosimetry for food irradiation. En: Johnston, D. E. \& Stevenson, M. H. Food Irradiation and the Chemist. Conference. Cambridge: Royal Society of Chemistry, pp. 109-123.

Silva, H. A., NunEs, M. L. y EmPIs, J. M. A. (1994): Sensory and microbiological assessment of irradiated bluejack mackerel (Trachurus picturatus): J. Sci. Food Agric. 66, 175-180.

SLOAN, A.E. (1999): Top trends to watch and work on for the millenium. Food Technology, 53(8), 40-60.

Tatsumi, Y., Chachin, K. y Ogata, K. (1974): Studies on the browning of potato tubers by gamma radiation. IV. Food Irradiation 9(12), 74-75.

Tatsumi, Y., Chachin, K. y Ogata, K. (1972): Studies on the browning of potatoes by gamma radiation. III. Food Irradiation 7(1), 82-83.

TEufEL, P. (1983): Microbiological aspects of food irradiation. En: Elias, P.S. y Cohen, A.J.(Ed.), Recent advances in food irradiation. Amsterdam: Elsevier Biomedical Press., pp. 217-234.

ThaYer, D. W. (1990): Food irradiation: benefits and concerns. Journal of Food Quality $13,147-169$.

THAYER, D. W. (1993): Extending shelf life of poultry and read meat by irradiation processing. J. Food Protect. 56(10), 831-833.

ThaYer, D. W. (1994): Wholesomeness of irradiated foods. Food Tech. 5, 132-135.

Thomas, P. (1986): Radiation preservation of foods of plant origin. III. Tropical fruits: bananas, mangoes, and papayas. CRC Critical Reviews in Food Science and $\mathrm{Nu}$ trition 23(2), 147-205. 